\title{
The Existence of Concatenated Codes List-Decodable up to the Hamming Bound*
}

\author{
Venkatesan Guruswami ${ }^{\dagger}$ \\ Computer Science Department \\ Carnegie Mellon University \\ Pittsburgh, PA 15213. \\ guruswami@cmu . edu \\ ATRI RudrA $\ddagger$ \\ Department of Computer Science and Engineering \\ University at Buffalo, The State University of New York \\ Buffalo, NY 14260. \\ atri@buffalo.edu
}

\begin{abstract}
We prove that binary linear concatenated codes with an outer algebraic code (specifically, a folded Reed-Solomon code) and independently and randomly chosen linear inner codes achieve, with high probability, the optimal trade-off between rate and list-decoding radius. In particular, for any $0<\rho<1 / 2$ and $\varepsilon>0$, there exist concatenated codes of rate at least $1-H(\rho)-\varepsilon$ that are (combinatorially) list-decodable up to a $\rho$ fraction of errors. (The Hamming bound states that the best possible rate for such codes cannot exceed $1-H(\rho)$, and standard random coding arguments show that this bound is approached by random codes with high probability.) A similar result, with better list size guarantees, holds when the outer code is also randomly chosen. Our methods and results extend to the case when the alphabet size is any fixed prime power $q \geqslant 2$.

Our result shows that despite the structural restriction imposed by code concatenation, the family of concatenated codes is rich enough to include codes list-decodable up to the optimal Hamming bound. This provides some encouraging news for tackling the problem of constructing explicit binary list-decodable codes with optimal rate, since code concatenation has been the preeminent method for constructing good codes for list decoding over small alphabets.
\end{abstract}

\footnotetext{
* A preliminary version of this paper appeared in the Proceedings of the Nineteenth Annual ACM-SIAM Symposium on Discrete Algorithms (SODA08) under the title "Concatenated codes can achieve list decoding capacity."

${ }^{\dagger}$ Research supported in part by a Packard Fellowship and NSF CCF-0953155.

${ }^{\ddagger}$ This work was done while the author was at the University of Washington and supported by NSF CCF-0343672.
} 


\section{Introduction}

A binary code $C \subseteq\{0,1\}^{n}$ is said to be $(\rho, L)$-list decodable if every Hamming ball of radius $\rho n$ in $\{0,1\}^{n}$ has at most $L$ codewords of $C$. Equivalently, if we pack Hamming balls of radius $\rho n$ centered at the codewords, no point in $\{0,1\}^{n}$ is covered more than $L$ times. Such a code $C$ enables correction of an arbitrary pattern of $\rho n$ errors in the model of list decoding, where the decoder is allowed to output a list of $L$ candidate codewords that must include the correct codeword. Here $L$ is the (output) "list size," which we typically think of as a constant independent of $n$ or a polynomially growing function of the block length $n$. When the exact list size is not important (beyond the fact that it is polynomially bounded in the block length), we will refer to $C$ as being list-decodable up to a fraction $\rho$ of errors or having list decoding radius $\rho$.

By the Hamming bound, the size of a $(\rho, L)$-list-decodable code satisfies $|C| \cdot \operatorname{Vol}_{2}(\rho, n) \leqslant 2^{n} \cdot L$ where $\operatorname{Vol}_{2}(\rho, n)=\sum_{i=0}^{\rho n}\left(\begin{array}{c}n \\ i\end{array}\right) \approx 2^{H(\rho) n}$ is the volume of the Hamming ball of radius $\rho n$ in $\{0,1\}^{n}$. Therefore, the rate of a binary code with list decoding radius $\rho$ is at most $1-H(\rho)+o(1)$.

Perhaps surprisingly, the simplistic Hamming upper bound on rate can in fact be achieved. In fact, a random code $C \subseteq\{0,1\}^{n}$ of rate $(1-H(\rho)-\varepsilon)$, obtained by picking $2^{(1-H(\rho)-\varepsilon) n}$ codewords randomly and independently, is $(\rho, 1 / \varepsilon)$-list decodable with high probability [18, 3]. We note that the choice of binary alphabet in this discussion is only for definiteness. Over an alphabet size $q \geqslant 2$, the Hamming bound on rate for a list decoding radius of $\rho$ equals $1-H_{q}(\rho)$, and random codes approach this trade-off with high probability (here $H_{q}(\cdot)$ is the $q$-ary entropy function). In the sequel, we will use the phrase "list-decodable up to the Hamming bound" to refer to codes that have rate $R$ and are list-decodable up to a fraction $H_{q}^{-1}(1-R)-\varepsilon$ of errors for any desired $\varepsilon>0$.

Unfortunately, the above is a nonconstructive argument and the codes list-decodable up to the Hamming bound are shown to exist by a random coding argument, and are not even succinctly, let alone explicitly, specified. It can also be shown that random linear codes are also list-decodable up to the Hamming bound w.h.p. However, till recently the known proofs of this only achieved a list size of $q^{O(1 / \varepsilon)}$ when the rate is $1-H_{q}(\rho)-\varepsilon[18$. (compared to the $O(1 / \varepsilon)$ list size bound known for general random codes), except for the case binary codes, where it was shown in [7 that a list size of $O(1 / \varepsilon)$ suffices (though the latter result was not shown to hold with high probability). In a recent work [6], it was shown that a random linear code over a field of size $q$ of rate $1-H_{q}(\rho)-\varepsilon$ is $\left(\rho, C_{\rho, q} / \varepsilon\right)$-list decodable with high probability. The advantage with linear codes is that they can be described succinctly via a generator or parity check matrix. Yet, a generic linear code offers little in terms of algorithmically useful structure, and in general only brute-force decoders running in exponential time are known for such a code.

Turning to constructive results for list decoding, recently explicit codes of rate $R$ and list decoding radius $1-R-\varepsilon$ together with polynomial time list-decoding algorithms up to this radius were constructed over large alphabets [10]. Using these as outer codes in a concatenation scheme led to polynomial time constructions of binary codes that achieved a rate vs. list-decoding radius trade-off called the Zyablov bound [10]. By using a multilevel generalization of code concatenation, the trade-off was recently improved to the so-called Blokh-Zyablov bound 11. Still, these explicit constructions fall well short of approaching the Hamming bound for binary (and other small alphabet) codes. Finding such a construction remains a major open problem. 
Concatenated codes and motivation behind this work. Ever since its discovery and initial use by Forney 4, code concatenation has been a powerful tool for constructing error-correcting codes. By concatenating an outer Reed-Solomon code of high rate with short inner codes achieving Shannon capacity (known to exist by a random coding argument), Forney 4 gave a construction of binary linear codes that achieve the capacity of the binary symmetric channel with a polynomial time decoding complexity. In comparison, Shannon's nonconstructive proof of his capacity theorem used an exponential time maximum likelihood decoder. Code concatenation was also the basis of Justesen's celebrated explicit construction of asymptotically good binary codes [13, where he used varying inner codes (from an explicit ensemble attaining the Gilbert-Varshamov bound) to encode different symbols of an outer Reed-Solomon code. For the longest time, till the work on expander codes by Sipser and Spielman [15, code concatenation schemes gave the only known explicit construction of a family of asymptotically good codes. Even today, the best trade-offs between rate and distance for explicit codes are achieved by variants of concatenated codes; see [2] for further details.

The same story applies to list decoding, where concatenated codes have been the preeminent and essentially only known method to construct codes achieving good trade-offs between rate and list decoding radius over small alphabets. Given the almost exclusive stronghold of concatenated codes on progress in explicit constructions of list-decodable codes over small alphabets, the following natural question arises: Do there exist binary concatenated codes that are list-decodable up to the Hamming bound, or does the stringent structural restriction imposed on the code by concatenation preclude achieving this?

The natural way to analyze the list decodability of concatenated codes suggests that perhaps concatenation is too strong a structural bottleneck to yield codes list-decodable up to the Hamming bound. Such an analysis proceeds by decoding the blocks of the received word corresponding to various inner encodings, which results in a small set $S_{i}$ of possible symbols for each position $i$ of the outer code. One then argues that there cannot be too many outer codewords whose $i$ 'th symbol belongs to $S_{i}$ for many positions $i$ (this is called a "list recovery" bound) 1 Even assuming optimal bounds on the individual list-decodability of the outer and inner codes, the above "two-stage" analysis bottlenecks at the Zyablov bound 2

The weakness of the two-stage analysis is that it treats the different inner decodings independently, and fails to exploit the fact that the various inner blocks encode a structured set of symbols, namely those arising in a codeword of the outer code. Exploiting this and arguing that the structure of the outer codewords prevents many "bad" inner blocks from occurring simultaneously, and using this to get improved bounds, however, seems like an intricate task. In part this is because the current understanding of "bad list-decoding configurations," i.e., Hamming balls of small radius containing many codewords, for codes is rather poor.

Our Results. In this work, we prove that there exist binary (and $q$-ary for any fixed prime power $q$ ) linear concatenated codes of any desired rate that are list-decodable up to the Hamming

\footnotetext{
${ }^{1}$ When the outer code is algebraic such as Reed-Solomon or folded Reed-Solomon, the list recovery step admits an efficient algorithm which leads to a polynomial time list-decoding algorithm for the concatenated code, such as in 10] 11 .

${ }^{2}$ One can squeeze out a little more out of the argument and achieve the Blokh-Zyablov bound, by exploiting the fact that sub-codes of the inner codes, being of lower rate, can be list decoded to a larger radius [11].
} 
bound. In fact, we prove that a random concatenated code drawn from a certain ensemble has such a list-decodability property with overwhelming probability. This is encouraging news for the eventual goal of explicitly constructing such codes (or at least, going beyond the above-mentioned Blokh-Zyablov bottleneck) over small alphabets.

The outer codes in our construction are the folded Reed-Solomon codes which were shown in [10] to have near-optimal list-recoverability properties 3 The inner codes for the various positions are random linear codes (which can even have a rate of 1), with a completely independent random choice for each outer codeword position. For a list decoding radius within $\varepsilon$ of the Hamming bound, our result guarantees an output list size bound that is a large polynomial (greater than $N^{1 / \varepsilon}$ ) in the block length $N$. We also prove that one can approach the Hamming bound on list decoding radius when a random linear code is chosen for the outer code; we get a better list size upper bound of a constant depending only on $\varepsilon$ in this case. A corollary of our result is that one can construct binary codes list-decodable up to the Hamming bound with a number of random bits that grows quasi-linearly in the block length, compared to the quadratic bound (achieved by a random linear code) known earlier.

Our results are inspired by results of Blokh and Zyablov [1] and Thommesen [16 showing the existence of binary concatenated codes whose rate vs. distance trade-off meets the GilbertVarshamov $(\mathrm{GV})$ bound. We recall that the GV bound is the best known trade-off between rate and relative distance for binary (and $q$-ary for $q<49$ ) codes and is achieved w.h.p. by random linear codes. Blokh and Zyablov show the result for independent random choices for the outer code and the various inner encodings. Thommesen establishes that one can fix the outer code to be a Reed-Solomon code and only pick the inner codes randomly (and independently).

Organization of the paper. Section 2 establishes the necessary background needed for the subsequent sections. We give a high level overview of our proof and how it compares with Thommesen's proof in Section 3. We present our results for concatenated codes with folded Reed-Solomon and random linear codes as outer codes in Sections 4 and 5 respectively. We conclude with some open questions in Section 6 .

\section{Preliminaries}

For an integer $m \geqslant 1$, we will use $[m]$ to denote the set $\{1, \ldots, m\}$.

\section{$2.1 \quad q$-ary Entropy and Related Functions}

Let $q \geqslant 2$ be an integer. $H_{q}(x)=x \log _{q}(q-1)-x \log _{q} x-(1-x) \log _{q}(1-x)$ will denote the $q$-ary entropy function. We will also need the inverse of the entropy function. In particular, for any $0 \leqslant y \leqslant 1$, define $H_{q}^{-1}(y)$ to be the unique value $x \in[0,1-1 / q]$ such that $H_{q}(x)=y$. We will make use of the following property of this function.

\footnotetext{
${ }^{3}$ We note that the excellent list-recoverability of folded Reed-Solomon codes is crucial for our argument, and we do not know how to prove a similar result using just Reed-Solomon codes as outer codes.
} 
Lemma 1 ([14]). For every $0 \leqslant y \leqslant 1$ and for every small enough $\varepsilon>0$, we have $H_{q}^{-1}\left(y-\varepsilon^{2} / c_{q}^{\prime}\right) \geqslant$ $H_{q}^{-1}(y)-\varepsilon$, where $c_{q}^{\prime} \geqslant 1$ is a constant that depends only on $q$.

For $0 \leqslant z \leqslant 1$ define

$$
\alpha_{q}(z)=1-H_{q}\left(1-q^{z-1}\right) .
$$

We will need the following property of the function above.

Lemma 2. Let $q \geqslant 2$ be an integer. For every $0 \leqslant z \leqslant 1, \alpha_{q}(z) \leqslant z$.

Proof. The proof follows from the subsequent sequence of relations:

$$
\begin{aligned}
\alpha_{q}(z) & =1-H_{q}\left(1-q^{z-1}\right) \\
& =1-\left(1-q^{z-1}\right) \log _{q}(q-1)+\left(1-q^{z-1}\right) \log _{q}\left(1-q^{z-1}\right)+q^{z-1}(z-1) \\
& =z q^{z-1}+\left(1-q^{z-1}\right)\left(1-\log _{q}\left(\frac{q-1}{1-q^{z-1}}\right)\right) \\
& \leqslant z
\end{aligned}
$$

where the last inequality follows from the facts that $q^{z-1} \leqslant 1$ and $1-q^{z-1} \leqslant 1-1 / q$, which implies that $\log _{q}\left(\frac{q-1}{1-q^{z-1}}\right) \geqslant 1$.

We will also consider the following function

$$
f_{x, q}(\theta)=(1-\theta)^{-1} \cdot H_{q}^{-1}(1-\theta x),
$$

where $0 \leqslant \theta, x \leqslant 1$. We will need the following property of this function, which was proven in [16] for the $q=2$ case. The following is an easy extension of the result for general $q$. (The main geometric intuition for $q>2$ also appears in [17].) For the sake of completeness, we provide a proof in Appendix $\mathrm{A}$

Lemma 3 ([16]). Let $q \geqslant 2$ be an integer. For any $x \geqslant 0$ and $0 \leqslant y \leqslant \alpha_{q}(x) / x$,

$$
\min _{0 \leqslant \theta \leqslant y} f_{x, q}(\theta)=(1-y)^{-1} H_{q}^{-1}(1-x y) .
$$

\subsection{Basic Coding Definitions}

A code of dimension $k$ and block length $n$ over an alphabet $\Sigma$ is a subset of $\Sigma^{n}$ of size $|\Sigma|^{k}$. The rate of such a code equals $k / n$. Each vector in $C$ is called a codeword. In this paper, we will focus on the case when $\Sigma$ is a finite field. We will denote by $\mathbb{F}_{q}$ the field with $q$ elements. A code $C$ over $\mathbb{F}_{q}$ is called a linear code if $C$ is a subspace of $\mathbb{F}_{q}^{n}$. In this case the dimension of the code coincides with the dimension of $C$ as a vector space over $\mathbb{F}_{q}$. By abuse of notation we will also think of a code $C$ as a map from elements in $\mathbb{F}_{q}^{k}$ to their corresponding codeword in $\mathbb{F}_{q}^{n}$. If $C$ is linear, this map is a linear transformation, mapping a row vector $x \in \mathbb{F}_{q}^{k}$ to a vector $x G \in \mathbb{F}_{q}^{n}$ for a $k \times n$ matrix $G$ over $\mathbb{F}_{q}$ called the generator matrix.

The Hamming distance between two vectors in $\Sigma^{n}$ is the number of places they differ in. The (minimum) distance of a code $C$ is the minimum Hamming distance between any two pairs of distinct codewords from $C$. The relative distance is the ratio of the distance to the block length. 


\subsection{Code Concatenation}

Concatenated codes are constructed from two different kinds of codes that are defined over alphabets of different sizes. Say we are interested in a code over $\mathbb{F}_{q}$ (in this paper, we will always think of $q \geqslant 2$ as being a fixed constant). Then the outer code $C_{\text {out }}$ is defined over $\mathbb{F}_{Q}$, where $Q=q^{k}$ for some positive integer $k$ and has block length $N$. The second type of code, called the inner codes, which are denoted by $C_{i n}^{1}, \ldots, C_{i n}^{N}$ are defined over $\mathbb{F}_{q}$ and are each of dimension $k$ (note that the message space of $C_{i n}^{i}$ for all $i$ and the alphabet of $C_{\text {out }}$ have the same size). The concatenated code, denoted by $C=C_{\text {out }} \circ\left(C_{i n}^{1}, \ldots, C_{i n}^{N}\right)$, is defined as follows. Let the rate of $C_{\text {out }}$ be $R$ and let the block lengths of $C_{i n}^{i}$ be $n$ (for $1 \leqslant i \leqslant N$ ). Define $K=R N$ and $r=k / n$. The input to $C$ is a vector $\mathbf{m}=\left\langle m_{1}, \ldots, m_{K}\right\rangle \in\left(\mathbb{F}_{q}^{k}\right)^{K}$. Let $C_{\text {out }}(\mathbf{m})=\left\langle x_{1}, \ldots, x_{N}\right\rangle$. The codeword in $C$ corresponding to $\mathbf{m}$ is defined as follows

$$
C(\mathbf{m})=\left\langle C_{i n}^{1}\left(x_{1}\right), C_{i n}^{2}\left(x_{2}\right), \ldots, C_{i n}^{N}\left(x_{N}\right)\right\rangle .
$$

The outer code $C_{\text {out }}$ will either be a random linear code over $\mathbb{F}_{Q}$ or the folded Reed-Solomon code from [10]. In the case when $C_{\text {out }}$ is random, we will pick $C_{\text {out }}$ by selecting $K=R N$ vectors uniformly at random from $\mathbb{F}_{Q}^{N}$ to form the rows of the generator matrix. For every position $1 \leqslant i \leqslant N$, we will choose an inner code $C_{i n}^{i}$ to be a random linear code over $\mathbb{F}_{q}$ of block length $n$ and rate $r=k / n$. In particular, we will work with the corresponding generator matrices $\mathbf{G}_{i}$, where every $\mathbf{G}_{i}$ is a random $k \times n$ matrix over $\mathbb{F}_{q}$. All the generator matrices $\mathbf{G}_{i}$ (as well as the generator matrix for $C_{\text {out }}$, when we choose a random $C_{\text {out }}$ ) are chosen independently. This fact will be used crucially in our proofs.

Given the outer code $C_{\text {out }}$ and the inner codes $C_{i n}^{i}$, recall that for every codeword $\mathbf{u}=$ $\left(\mathbf{u}_{1}, \ldots, \mathbf{u}_{N}\right) \in C_{\text {out }}$, the codeword $\mathbf{u G} \stackrel{\text { def }}{=}\left(\mathbf{u}_{1} \mathbf{G}_{1}, \mathbf{u}_{2} \mathbf{G}_{2}, \ldots, \mathbf{u}_{N} \mathbf{G}_{N}\right)$ is in $C=C_{\text {out }} \circ\left(C_{\text {in }}^{1}, \ldots, C_{\text {in }}^{N}\right)$, where the operations are over $\mathbb{F}_{q}$.

We will need the following notions of the weight of a vector. Given a vector $\mathbf{v} \in \mathbb{F}_{q}^{n N}$, its Hamming weight is denoted by $w t(\mathbf{v})$. Given a vector $\mathbf{y}=\left(y_{1}, \ldots, y_{N}\right) \in\left(\mathbb{F}_{q}^{n}\right)^{N}$ and a subset $S \subseteq[N]$, we will use $w t_{S}(\mathbf{y})$ to denote the Hamming weight over $\mathbb{F}_{q}$ of the subvector $\left(y_{i}\right)_{i \in S}$. Note that $w t(\mathbf{y})=w t_{[N]}(\mathbf{y})$.

We will need the following simple lemma due to Thommesen, which is stated in a slightly different form in [16]. For the sake of completeness we also present its proof.

Lemma 4 (16]). Given a fixed outer code $C_{\text {out }}$ of block length $N$ and an ensemble of random inner linear codes of block length $n$ given by generator matrices $\mathbf{G}_{1}, \ldots, \mathbf{G}_{N}$ the following is true. Let $\mathbf{y} \in \mathbb{F}_{q}^{n N}$. For any codeword $\mathbf{u} \in C_{\text {out }}$, any non-empty subset $S \subseteq[N]$ such that $\mathbf{u}_{i} \neq 0$ for all $i \in S$ and any integer $h \leqslant n|S| \cdot\left(1-\frac{1}{q}\right)$ :

$$
\operatorname{Pr}\left[w t_{S}(\mathbf{u G}-\mathbf{y}) \leqslant h\right] \leqslant q^{-n|S|\left(1-H_{q}\left(\frac{h}{n|S|}\right)\right)}
$$

where the probability is taken over the random choices of $\mathbf{G}_{1}, \ldots, \mathbf{G}_{N}$.

Proof. Let $|S|=s$ and w.l.o.g. assume that $S=[s]$. As the choices for $\mathbf{G}_{1}, \ldots, \mathbf{G}_{N}$ are made independently, it is enough to show that the claimed probability holds for the random choices for $\mathbf{G}_{1}, \ldots, \mathbf{G}_{s}$. For any $1 \leqslant i \leqslant s$ and any $y \in \mathbb{F}_{q}^{n}$, since $\mathbf{u}_{i} \neq 0$, we have $\operatorname{Pr}_{\mathbf{G}_{i}}\left[\mathbf{u}_{i} \mathbf{G}_{i}=y\right]=q^{-n}$. 
Further, these probabilities are independent for every $i$. Thus, for any $\mathbf{y}=\left\langle y_{1}, \ldots, y_{s}\right\rangle \in\left(\mathbb{F}_{q}^{n}\right)^{s}$, $\operatorname{Pr}_{\mathbf{G}_{1}, \ldots, \mathbf{G}_{s}}\left[\mathbf{u}_{i} \mathbf{G}_{i}=y_{i}\right.$ for every $\left.1 \leqslant i \leqslant s\right]=q^{-n s}$. This implies that:

$$
\operatorname{Pr}_{\mathbf{G}_{1}, \ldots, \mathbf{G}_{s}}\left[w t_{S}(\mathbf{u G}-\mathbf{y}) \leqslant h\right]=q^{-n s} \sum_{j=0}^{h}\left(\begin{array}{c}
n s \\
j
\end{array}\right)(q-1)^{j} .
$$

The claimed result follows from the following well known inequality for $h /(n s) \leqslant 1-1 / q([12])$ :

$$
\sum_{j=0}^{h}\left(\begin{array}{c}
n s \\
j
\end{array}\right)(q-1)^{j} \leqslant q^{n s H_{q}\left(\frac{h}{n s}\right)} .
$$

\subsection{List Decoding and List Recovery}

Definition 1 (List decodable code). For $0<\rho<1$ and an integer $L \geqslant 1$, a code $C \subseteq \mathbb{F}_{q}^{n}$ is said to be $(\rho, L)$-list decodable if for every $y \in \mathbb{F}_{q}^{n}$, the number of codewords in $C$ that are within Hamming distance $\rho n$ from $y$ is at most $L$.

We will also crucially use a generalization of list decoding called list recovery, a term first coined in [8] even though the notion had existed before. List recovery has been extremely useful in listdecoding concatenated codes. The input for list recovery is not a sequence of symbols but rather a sequence of subsets of allowed codeword symbols, one for each codeword position.

Definition 2 (List recoverable code). A code $C \subseteq \mathbb{F}_{q}^{n}$, is called $(\rho, \ell, L)$-list recoverable if for every sequence of sets $S_{1}, S_{2}, \ldots, S_{n}$, where $S_{i} \subseteq \mathbb{F}_{q}$ and $\left|S_{i}\right| \leqslant \ell$ for every $1 \leqslant i \leqslant n$, there are at most $L$ codewords $\left(c_{1}, \ldots, c_{n}\right) \in C$ such that $c_{i} \in S_{i}$ for at least $(1-\rho)$ n positions $i$.

The classical family of Reed-Solomon (RS) codes over a field $\mathbb{F}$ are defined to be the evaluations of low-degree polynomials at a sequence of distinct points of $\mathbb{F}$. Folded Reed-Solomon codes are obtained by viewing the RS code as a code over a larger alphabet $\mathbb{F}^{s}$ by bundling together consecutive $s$ symbols for some folding parameter $s$. We will not need any specifics of folded RS codes (in fact even its definition) beyond (i) the strong list recovery property guaranteed by the following theorem from [10], and (ii) the fact that specifying any $K+1$ positions in a dimension $K$ folded Reed-Solomon code suffices to identify the codeword (equivalently, a dimension $K$ and length $N$ folded RS code has distance at least $N-K$ ).

Theorem 1 (10]). For every integer $\ell \geqslant 1$, for all constants $\varepsilon>0$, for all $0<R<1$, and for every prime $p$, there is an explicit family of folded Reed-Solomon codes, over fields of characteristic $p$ that have rate at least $R$ and which can be $(1-R-\varepsilon, \ell, L(N))$-list recovered in polynomial time, where for codes of block length $N, L(N)=\left(N / \varepsilon^{2}\right)^{O\left(\varepsilon^{-1} \log (\ell / R)\right)}$ and the code is defined over alphabet of size $\left(N / \varepsilon^{2}\right) O\left(\varepsilon^{-2} \log \ell /(1-R)\right)$.

\subsection{A Limited Independence Result}

We state a lemma that will be useful in our proofs later. 
Lemma 5. Let $q$ be a prime power and let $k, \ell \geqslant 1$ be integers. Let $n \geqslant k$ be an integer and let $\mathbf{v}_{1}, \ldots, \mathbf{v}_{\ell} \in \mathbb{F}_{q}^{k}$ be vectors such that $\mathbf{v}_{\ell}$ is not in the $\mathbb{F}_{q}$-span of $\left\{\mathbf{v}_{1}, \ldots, \mathbf{v}_{\ell-1}\right\}$. Then the following holds for every $\alpha_{1}, \ldots, \alpha_{\ell} \in \mathbb{F}_{q}^{n}$ :

$$
\underset{\mathbf{G}}{\operatorname{Pr}}\left[\bigwedge_{i=1}^{\ell} \mathbf{v}_{i} \mathbf{G}=\alpha_{i}\right]=\underset{\mathbf{G}}{\operatorname{Pr}}\left[\mathbf{v}_{\ell} \mathbf{G}=\alpha_{\ell}\right] \cdot \underset{\mathbf{G r}}{\operatorname{Pr}}\left[\bigwedge_{i=1}^{\ell-1} \mathbf{v}_{i} \mathbf{G}=\alpha_{i}\right],
$$

where $\mathbf{G}$ is a random $k \times n$ matrix over $\mathbb{F}_{q}$. In particular, the statement also holds if $\left\{\mathbf{v}_{1}, \ldots, \mathbf{v}_{\ell}\right\}$ are $\mathbb{F}_{q}$-linearly independent.

Proof. If $\left\{\mathbf{v}_{1}, \ldots, \mathbf{v}_{\ell}\right\}$ are $\mathbb{F}_{q}$-linearly independent, then it is well known that the vectors $\left\{\mathbf{v}_{1} \mathbf{G}, \ldots, \mathbf{v}_{\ell} \mathbf{G}\right\}$ are random independent vectors from $\mathbb{F}_{q}^{n}$ (for a proof see e.g. 11]). Thus, under this special condition, (2) is true. Next, we reduce the more general case to this special case.

First we claim we can reduce the general case to the case where for every $1 \leqslant i<\ell, \mathbf{v}_{i} \neq \mathbf{0}$. (Note that $\mathbf{v}_{\ell}$ is always non-zero.) To see this, w.l.o.g. assume that $\mathbf{v}_{1}=\mathbf{0}$. Now note that if $\alpha_{1} \neq \mathbf{0}$, then (2) is true as $\operatorname{Pr}_{\mathbf{G}}\left[\mathbf{v}_{1} \mathbf{G}=\alpha_{1}\right]=0$ (which in turn implies that $\operatorname{Pr}_{\mathbf{G}}\left[\wedge_{i=1}^{\ell} \mathbf{v}_{i} \mathbf{G}=\alpha_{i}\right]=$ $\left.\operatorname{Pr}_{\mathbf{G}}\left[\wedge_{i=1}^{\ell-1} \mathbf{v}_{i} \mathbf{G}=\alpha_{i}\right]=0\right)$. Now if $\alpha_{1}=\mathbf{0}$, then since $\operatorname{Pr}_{\mathbf{G}}\left[\mathbf{v}_{\mathbf{1}} \mathbf{G}=\alpha_{1}\right]=1$, which in turn implies that

$$
\underset{\mathbf{G}}{\operatorname{Pr}}\left[\bigwedge_{i=1}^{\ell} \mathbf{v}_{i} \mathbf{G}=\alpha_{i}\right]=\underset{\mathbf{G}}{\operatorname{Pr}}\left[\bigwedge_{i=2}^{\ell} \mathbf{v}_{i} \mathbf{G}=\alpha_{i}\right] \text { and } \underset{\mathbf{G}}{\operatorname{Pr}}\left[\bigwedge_{i=1}^{\ell-1} \mathbf{v}_{i} \mathbf{G}=\alpha_{i}\right]=\underset{\mathbf{G}}{\operatorname{Pr}}\left[\bigwedge_{i=2}^{\ell-1} \mathbf{v}_{i} \mathbf{G}=\alpha_{i}\right] .
$$

Applying the argument above inductively on all the $\mathbf{0}$ vectors among $\mathbf{v}_{1}, \ldots, \mathbf{v}_{\ell-1}$, we can get rid of all such vectors.

Thus, from now on we will assume that $\mathbf{v}_{i} \neq \mathbf{0}$ for every $1 \leqslant i \leqslant \ell$. To complete the proof, we show how to reduce this case to the case where $\left\{\mathbf{v}_{1}, \ldots, \mathbf{v}_{\ell-1}\right\}$ are all linearly independent. (Note that in the latter case $\left\{\mathbf{v}_{1}, \ldots, \mathbf{v}_{\ell}\right\}$ are $\mathbb{F}_{q}$-linearly independent and by the earlier argument, we will be done.) W.l.o.g. assume that $\left\{\mathbf{v}_{1}, \ldots, \mathbf{v}_{t}\right\}$ is a maximum set of $\mathbb{F}_{q}$-linearly independent vectors among $\left\{\mathbf{v}_{1}, \ldots, \mathbf{v}_{\ell-1}\right\}$ for some $1 \leqslant t<\ell$. Note that this implies that once the values of $\mathbf{v}_{1} \mathbf{G}, \ldots, \mathbf{v}_{t} \mathbf{G}$ are fixed, then so are the values of $\mathbf{v}_{t+1} \mathbf{G}, \ldots, \mathbf{v}_{\ell-1} \mathbf{G}$. Call $\alpha_{1}, \ldots, \alpha_{\ell-1}$ consistent, if the values $\alpha_{t+1}, \ldots, \alpha_{\ell-1}$ are what they should be given $\alpha_{1}, \ldots, \alpha_{t}$. (Otherwise call the values inconsistent.) It is easy to see that if $\alpha_{1}, \ldots, \alpha_{\ell-1}$ are inconsistent then (2) is true as both the LHS and RHS are 0 . On the other hand if $\alpha_{1}, \ldots, \alpha_{\ell-1}$ are consistent, then we have

$$
\underset{\mathbf{G}}{\operatorname{Pr}}\left[\bigwedge_{i=1}^{\ell-1} \mathbf{v}_{i} \mathbf{G}=\alpha_{i}\right]=\underset{\mathbf{G}}{\operatorname{Pr}}\left[\bigwedge_{i=1}^{t} \mathbf{v}_{i} \mathbf{G}=\alpha_{i}\right] \text { and } \underset{\mathbf{G}}{\operatorname{Pr}}\left[\bigwedge_{i=1}^{\ell} \mathbf{v}_{i} \mathbf{G}=\alpha_{i}\right]=\underset{\mathbf{G}}{\operatorname{Pr}}\left[\mathbf{v}_{\ell} \mathbf{G}=\alpha_{\ell} \wedge \bigwedge_{i=1}^{t} \mathbf{v}_{i} \mathbf{G}=\alpha_{i}\right] \text {, }
$$

which completes the proof.

\section{Overview of the Proof}

Our proofs are inspired by Thommesen's proof [16] of the following result concerning the rate vs. distance trade-off of concatenated codes: Binary linear concatenated codes with an outer ReedSolomon code and independently and randomly chosen inner codes meet the Gilbert-Varshamov 
bound with high probability 4 , provided a moderate condition on the outer and inner rates is met. Given that our proof builds on the proof of Thommesen, we start out by reviewing the main ideas in his proof.

The outer code $C_{\text {out }}$ in [16] is a Reed-Solomon code of length $N$ and rate $R$ (over $\mathbb{F}_{Q}$ where $Q=q^{k}$ for some integer $k \geqslant 1$ ). The inner linear codes (over $\mathbb{F}_{q}$ ) are generated by $N$ randomly chosen $k \times n$ generator matrices $\mathbf{G}=\left(\mathbf{G}_{1}, \ldots, \mathbf{G}_{N}\right)$, where $r=k / n$. Note that since the final code will be linear, to show that with high probability the concatenated code will have distance close to $H_{q}^{-1}(1-r R)$, it is enough to show that the probability of the Hamming weight of $\mathbf{u G}$ over $\mathbb{F}_{q}$ being at most $\left(H_{q}^{-1}(1-r R)-\varepsilon\right) n N$ (for every non-zero Reed-Solomon codeword $\mathbf{u}=\left(\mathbf{u}_{1}, \ldots, \mathbf{u}_{N}\right)$ and $\varepsilon>0$ ), is small. Fix a codeword $\mathbf{u} \in C_{\text {out }}$. Now note that if for some $1 \leqslant i \leqslant N, \mathbf{u}_{i}=0$, then for every choice of $\mathbf{G}_{i}, \mathbf{u}_{i} \mathbf{G}_{i}=0$. Thus, only the non-zero symbols of $\mathbf{u}$ contribute to $w t(\mathbf{u G})$. Further, for a non-zero $\mathbf{u}_{i}, \mathbf{u}_{i} \mathbf{G}_{i}$ takes all the values in $\mathbb{F}_{q}^{n}$ with equal probability over the random choices of $\mathbf{G}_{i}$. Since the choice of the $\mathbf{G}_{i}$ 's are independent, this implies that $\mathbf{u G}$ takes each of the possible $q^{n \cdot w t(\mathbf{u})}$ values in $\mathbb{F}_{q}^{n N}$ with the same probability. Thus, the total probability that $\mathbf{u G}$ has a Hamming weight of at most $h$ is

$$
\sum_{w=0}^{h}\left(\begin{array}{c}
n \cdot w t(\mathbf{u}) \\
w
\end{array}\right) q^{-n \cdot w t(\mathbf{u})} \leqslant q^{-n \cdot w t(\mathbf{u})\left(1-H_{q}\left(\frac{h}{n \cdot w t(\mathbf{u})}\right)\right)}
$$

(this is Lemma 4 for the case $S=\left\{i \mid \mathbf{u}_{i} \neq 0\right\}$ and $\mathbf{y}=\mathbf{0}$ ). The rest of the argument follows by doing a careful union bound of this probability for all non zero codewords in $C_{\text {out }}$, using the weight distribution of the RS code. This step imposes an upper bound on the outer rate $R$ (specifically, $\left.R \leqslant \alpha_{q}(r) / r\right)$, but still offers enough flexibility to achieve any desired value in $(0,1)$ for the overall rate $r R$ (even with the choice $r=1$, i.e., when the inner encodings don't add any redundancy).

Let us now try to extend the idea above to show a similar result for list decoding. We want to show that for any Hamming ball of radius at most $h=\left(H_{q}^{-1}(1-r R)-\varepsilon\right) n N$ has at most $L$ codewords from the concatenated code $C$ (assuming we want to show that $L$ is the worst case list size). To show this let us look at a set of $L+1$ codewords from $C$ and try to prove that the probability that all of them lie within some fixed ball $\mathcal{B}$ of radius $h$ is small. Let $\mathbf{u}^{1}, \ldots, \mathbf{u}^{L+1}$ be the corresponding codewords in $C_{\text {out }}$. Extending Thommesen's proof would be straightforward if the events corresponding to $\mathbf{u}^{j} \mathbf{G}$ belonging to the ball $\mathcal{B}$ for various $1 \leqslant j \leqslant L+1$ were independent. In particular, if we can show that for every position $1 \leqslant i \leqslant N$, all the non-zero symbols in $\left\{\mathbf{u}_{i}^{1}, \mathbf{u}_{i}^{2}, \ldots, \mathbf{u}_{i}^{L+1}\right\}$ are linearly independent over $\mathbb{F}_{q}$ then the generalization of Thommesen's proof is immediate.

Unfortunately, the notion of independence discussed above does not hold for every $L+1$ tuple of codewords from $C_{\text {out }}$. The natural way to get independence when dealing with linear codes is to look at messages that are linearly independent. It turns out that if $C_{\text {out }}$ is also a random linear code over $\mathbb{F}_{Q}$ then we have a good approximation of the the notion of independence above. Specifically, we show that with very high probability for a linearly independent (over $\mathbb{F}_{Q}$ ) set of message $5 \mathbf{m}^{1}, \ldots, \mathbf{m}^{L+1}$, the set of codewords $\mathbf{u}^{1}=C_{\text {out }}\left(\mathbf{m}^{1}\right), \ldots, \mathbf{u}^{L+1}=C_{\text {out }}\left(\mathbf{m}^{L+1}\right)$ have the following approximate independence property. For many positions $1 \leqslant i \leqslant N$, many non-zero

\footnotetext{
${ }^{4} \mathrm{~A} q$-ary code of rate $\mathcal{R}$ meets the Gilbert-Varshamov bound if it has relative distance at least $H_{q}^{-1}(1-\mathcal{R})$.

${ }^{5}$ Again any set of $L+1$ messages need not be linearly independent. However, it is easy to see that some subset of $J=\left\lceil\log _{Q}(L+1)\right\rceil$ of messages are indeed linearly independent. Hence, we can continue the argument by replacing $L+1$ with $J$.
} 
symbols in $\left\{\mathbf{u}_{i}^{1}, \ldots, \mathbf{u}_{i}^{L+1}\right\}$ are linearly independent over $\mathbb{F}_{q}$. It turns out that this approximate notion of independence is enough for Thommesen's proof to go through.

We remark that the notion above crucially uses the fact that the outer code is a random linear code. The argument gets more tricky when $C_{\text {out }}$ is fixed to be (say) the Reed-Solomon code. Now even if the messages $\mathbf{m}^{1}, \ldots, \mathbf{m}^{L+1}$ are linearly independent it is not clear that the corresponding codewords will satisfy the notion of independence in the above paragraph. Interestingly, we can show that this notion of independence is equivalent to showing good list recoverability properties for $C_{\text {out }}$. Reed-Solomon codes are however not known to have optimal list recoverability (which is what is required in our case). In fact, the results in [9] show that this is impossible for ReedSolomon codes in general. However, folded RS codes do have near-optimal list recoverability and we exploit this in our proof.

\section{Using Folded Reed-Solomon Code as Outer Code}

In this section, we will prove that concatenated codes with the outer code being the folded ReedSolomon code from [10] and using random and independent inner codes can achieve list-decoding capacity. The proof will make crucial use of the list recoverability of the outer code as stated in Theorem 1 .

\subsection{Linear Independence from List Recoverability}

Definition 3 (Independent tuples). Let $C$ be a code of block length $N$ and rate $R$ defined over $\mathbb{F}_{q^{k}}$. Let $J \geqslant 1$ and $0 \leqslant d_{1}, \ldots, d_{J} \leqslant N$ be integers. Let $\mathbf{d}=\left\langle d_{1}, \ldots, d_{J}\right\rangle$. An ordered tuple of codewords $\left(c^{1}, \ldots, c^{J}\right), c^{j} \in C$ is said to be $\left(\mathbf{d}, \mathbb{F}_{q}\right)$-independent if the following holds. $d_{1}=w t\left(c^{1}\right)$ and for every $1<j \leqslant J, d_{j}$ is the number of positions $i$ such that $c_{i}^{j}$ is not in the $\mathbb{F}_{q}$-span of the vectors $\left\{c_{i}^{1}, \ldots, c_{i}^{j-1}\right\}$, where $c^{\ell}=\left(c_{1}^{\ell}, \ldots, c_{N}^{\ell}\right)$.

Note that for any tuple of codewords $\left(c^{1}, \ldots, c^{J}\right)$ there exists a unique $\mathbf{d}$ such that it is $\left(\mathbf{d}, \mathbb{F}_{q}\right)$ independent. The next result will be crucial in our proof.

Lemma 6. Let $C$ be a folded Reed-Solomon code of block length $N$ that is defined over $\mathbb{F}_{Q}$ with $Q=q^{k}$ as guaranteed by Theorem [1. For any L-tuple of codewords from $C$, where $L \geqslant J$. $\left(N / \varepsilon^{2}\right)^{O\left(\varepsilon^{-1} J \log (q / R)\right)}$ (where $\varepsilon>0$ is same as the one in Theorem 1), there exists a sub-tuple of $J$ codewords such that the $J$-tuple is $\left(\mathbf{d}, \mathbb{F}_{q}\right)$-independent, where $\mathbf{d}=\left\langle d_{1}, \ldots, d_{J}\right\rangle$ with $d_{j} \geqslant$ $(1-R-\varepsilon) N$, for every $1 \leqslant j \leqslant J$.

Proof. The proof is constructive. In particular, given an $L$-tuple of codewords, we will construct a $J$ sub-tuple with the required property. The correctness of the procedure will hinge on the list recoverability of the folded Reed-Solomon code as guaranteed by Theorem 1 .

We will construct the final sub-tuple iteratively. In the first step, pick any non-zero codeword in the $L$-tuple- call it $c^{1}$. As $C$ has distance at least $(1-R) N$ (and $\mathbf{0} \in C$ ), $c^{1}$ is non-zero in at least $d_{1} \geqslant(1-R) N>(1-R-\varepsilon) N$ many places. Note that $c^{1}$ is vacuously not in the span of the "previous" codewords in these positions. Now, say that the procedure has chosen codewords $c^{1}, \ldots, c^{s}$ such that the tuple is $\left(\mathbf{d}^{\prime}, \mathbb{F}_{q}\right)$-independent for $\mathbf{d}^{\prime}=\left\langle d_{1}, \ldots, d_{s}\right\rangle$, where for every $1 \leqslant j \leqslant s$, 
$d_{j} \geqslant(1-R-\varepsilon) N$. For every $1 \leqslant i \leqslant N$, define $S_{i}$ to be the $\mathbb{F}_{q^{-}}$span of the vectors $\left\{c_{i}^{1}, \ldots, c_{i}^{s}\right\}$ in $\mathbb{F}_{q}^{k}$. Note that $\left|S_{i}\right| \leqslant q^{s}$. Call $c=\left(c_{1}, \ldots, c_{N}\right) \in C$ to be a bad codeword, if there does not exist any $d_{s+1} \geqslant(1-R-\varepsilon) N$ such that $\left(c^{1}, \ldots, c^{s}, c\right)$ is $\left(\mathbf{d}, \mathbb{F}_{q}\right)$-independent for $\mathbf{d}=\left\langle d_{1}, \ldots, d_{s+1}\right\rangle$. In other words, $c$ is a bad codeword if and only if some $T \subset[N]$ with $|T|=(R+\varepsilon) N$ satisfies $c_{i} \in S_{i}$ for every $i \in T$. Put differently, $c$ satisfies the condition of being in the output list for list recovering $C$ with input $S_{1}, \ldots, S_{N}$ and agreement fraction $R+\varepsilon$. Thus, by Theorem 1 the number of such bad codewords is $U=\left(N / \varepsilon^{2}\right)^{O\left(\varepsilon^{-1} s \log (q / R)\right)} \leqslant\left(N / \varepsilon^{2}\right)^{O\left(\varepsilon^{-1} J \log (q / R)\right)}$, where $J$ is the number of steps for which this greedy procedure can be applied. Thus, as long as at each step there are strictly more than $U$ codewords from the original $L$-tuple of codewords left, we can continue this greedy procedure. Note that we can continue this procedure $J$ times, as long as $J \leqslant L / U$.

Finally, we will need the following bound on the number of independent tuples for folded ReedSolomon codes. Its proof follows from the fact that a codeword in a dimension $K$ folded RS code is completely determined once values at $K+1$ of its positions are fixed.

Lemma 7. Let $C$ be a folded Reed-Solomon code of block length $N$ and rate $0<R<1$ that is defined over $\mathbb{F}_{Q}$, where $Q=q^{k}$. Let $J \geqslant 1$ and $0 \leqslant d_{1}, \ldots, d_{J} \leqslant N$ be integers and define $\mathbf{d}=\left\langle d_{1}, \ldots, d_{J}\right\rangle$. Then the number of $\left(\mathbf{d}, \mathbb{F}_{q}\right)$-independent tuples in $C$ is at most

$$
q^{N J(J+1)} \prod_{j=1}^{J} Q^{\max \left(d_{j}-N(1-R)+1,0\right)} .
$$

Proof. Given a tuple $\left(c^{1}, \ldots, c^{J}\right)$ that is $\left(\mathbf{d}, \mathbb{F}_{q}\right)$-independent, define $T_{j} \subseteq[N]$ with $\left|T_{j}\right|=d_{j}$, for $1 \leqslant j \leqslant J$ to be the set of positions $i$, where $c_{i}^{j}$ is not in the $\mathbb{F}_{q^{-}}$span of $\left\{c_{i}^{1}, \ldots, c_{i}^{j-1}\right\}$. We will estimate the number of $\left(\mathbf{d}, \mathbb{F}_{q}\right)$-independent tuples by first estimating a bound $U_{j}$ on the number of choices for the $j^{\text {th }}$ codeword in the tuple (given a fixed choice of the first $j-1$ codewords). To complete the proof, we will show that

$$
U_{j} \leqslant q^{N(J+1)} \cdot Q^{\max \left(d_{j}-N(1-R)+1,0\right)} .
$$

A codeword $c \in C$ can be the $j^{\text {th }}$ codeword in the tuple in the following way. For every position in $[N] \backslash T_{j}, c$ can take at most $q^{j-1} \leqslant q^{J}$ values (as in these position the value has to lie in the $\mathbb{F}_{q}$ span of the values of the first $j-1$ codewords in that position). Since $C$ is folded Reed-Solomon, once we fix the values at positions in $[N] \backslash T_{j}$, the codeword will be completely determined once any $\max \left(R N-\left(N-d_{j}\right)+1,0\right)=\max \left(d_{j}-N(1-R)+1,0\right)$ positions in $T_{j}$ are chosen (w.l.o.g. assume that they are the "first" so many positions). The number of choices for $T_{j}$ is $\left(\begin{array}{l}N \\ d_{j}\end{array}\right) \leqslant 2^{N} \leqslant q^{N}$. Thus, we have

$$
\begin{aligned}
U_{j} & \leqslant q^{N} \cdot\left(q^{J}\right)^{N-d_{j}} \cdot Q^{\max \left(d_{j}-N(1-R)+1,0\right)} \\
& \leqslant q^{N(J+1)} \cdot Q^{\left.\max \left(d_{j}-N(1-R)+1\right), 0\right)},
\end{aligned}
$$

as desired.

\subsection{The Main Result}

Theorem 2 (Main). Let $q$ be a prime power and let $0<r \leqslant 1$ be an arbitrary rational. Let $0<\varepsilon<\alpha_{q}(r)$ an arbitrary real, where $\alpha_{q}(r)$ is as defined in (11), and $0<R \leqslant\left(\alpha_{q}(r)-\varepsilon\right) / r$ 
be a rational. Let $k, n, K, N \geqslant 1$ be large enough integers such that $k=r n$ and $K=R N$. Let $C_{\text {out }}$ be a folded Reed-Solomon code over $\mathbb{F}_{q^{k}}$ of block length $N$ and rate $R$. Let $C_{i n}^{1}, \ldots, C_{i n}^{N}$ be random linear codes over $\mathbb{F}_{q}$, where $C_{\text {in }}^{i}$ is generated by a random $k \times n$ matrix $\mathbf{G}_{i}$ over $\mathbb{F}_{q}$ and the random choices for $\mathbf{G}_{1}, \ldots, \mathbf{G}_{N}$ are all independent 6 Then the concatenated code $C=$ $C_{\text {out }} \circ\left(C_{\text {in }}^{1}, \ldots, C_{\text {in }}^{N}\right)$ is a $\left(H_{q}^{-1}(1-R r)-\varepsilon,\left(\frac{N}{\varepsilon^{4}}\right)^{O\left(r^{2} \varepsilon^{-4}(1-R)^{-2} \log (1 / R)\right)}\right)$-list decodable code with probability at least $1-q^{-\Omega(n N)}$ over the choices of $\mathbf{G}_{1}, \ldots, \mathbf{G}_{N}$. Further, $C$ has rate $r R$ w.h.p.

Remark 1. For any desired rate $R^{*} \in(0,1-\varepsilon)$ for the final concatenated code (here $\varepsilon>0$ is arbitrary), one can pick the outer and inner rates $R, r$ such that $R r=R^{*}$ while also satisfying $R \leqslant\left(\alpha_{q}(r)-\varepsilon\right) / r$. In fact we can pick $r=1$ and $R=R^{*}$ so that the inner encodings are linear transformations specified by random $k \times k$ matrices and do not add any redundancy.

The rest of this section is devoted to proving Theorem 2

Define $Q=q^{k}$. Let $L$ be the worst-case list size that we are shooting for (we will fix its value at the end). By Lemma 6] any $L+1$-tuple of $C_{\text {out }}$ codewords $\left(\mathbf{u}^{0}, \ldots, \mathbf{u}^{L}\right) \in\left(C_{\text {out }}\right)^{L+1}$ contains at least $J=\left\lfloor(L+1) /\left(N / \gamma^{2}\right)^{O\left(\gamma^{-1} J \log (q / R)\right)}\right\rfloor$ codewords that form an $\left(\mathbf{d}, \mathbb{F}_{q}\right)$-independent tuple, for some $\mathbf{d}=\left\langle d_{1}, \ldots, d_{J}\right\rangle$, with $d_{j} \geqslant(1-R-\gamma) N$ (we will specify $\gamma, 0<\gamma<1-R$, later). Thus, to prove the theorem it suffices to show that with high probability, no Hamming ball in $\mathbb{F}_{q}^{n N}$ of radius $\left(H_{q}^{-1}(1-r R)-\varepsilon\right) n N$ contains a $J$-tuple of codewords $\left(\mathbf{u}^{1} \mathbf{G}, \ldots, \mathbf{u}^{J} \mathbf{G}\right)$, where $\left(\mathbf{u}^{1}, \ldots, \mathbf{u}^{J}\right)$ is a $J$-tuple of folded Reed-Solomon codewords that is $\left(\mathbf{d}, \mathbb{F}_{q}\right)$-independent. For the rest of the proof, we will call a $J$-tuple of $C_{\text {out }}$ codewords $\left(\mathbf{u}^{1}, \ldots, \mathbf{u}^{J}\right)$ a good tuple if it is $\left(\mathbf{d}, \mathbb{F}_{q}\right)$-independent for some $\mathbf{d}=\left\langle d_{1}, \ldots, d_{J}\right\rangle$, where $d_{j} \geqslant(1-R-\gamma) N$ for every $1 \leqslant j \leqslant J$.

Define $\rho=H_{q}^{-1}(1-R r)-\varepsilon$. For every good $J$-tuple of $C_{\text {out }}$ codewords $\left(\mathbf{u}^{1}, \ldots, \mathbf{u}^{J}\right)$ and received word $\mathbf{y} \in \mathbb{F}_{q}^{n N}$, define an indicator variable $\mathbf{I}\left(\mathbf{y}, \mathbf{u}^{1}, \ldots, \mathbf{u}^{J}\right)$ as follows. $\mathbf{I}\left(\mathbf{y}, \mathbf{u}^{1}, \ldots, \mathbf{u}^{J}\right)=1$ if and only if for every $1 \leqslant j \leqslant J, w t\left(\mathbf{u}^{j} \mathbf{G}-\mathbf{y}\right) \leqslant \rho n N$. That is, it captures the bad event that we want to avoid. Define

$$
X_{C}=\sum_{\mathbf{y} \in \mathbb{F}_{q}^{n N} \operatorname{good}} \sum_{\left(\mathbf{u}^{1}, \ldots, \mathbf{u}^{J}\right) \in\left(C_{\text {out }}\right)^{J}} \mathbf{I}\left(\mathbf{y}, \mathbf{u}^{1}, \ldots, \mathbf{u}^{J}\right) .
$$

We want to show that with high probability $X_{C}=0$. By Markov's inequality, the theorem would follow if we can show that:

$$
\begin{aligned}
\mathbb{E}\left[X_{C}\right] & =\sum_{\mathbf{y} \in \mathbb{F}_{q}^{n N}} \sum_{\substack{\text { good } \\
\in\left(\mathbf{u}^{1}, \ldots, \mathbf{u}^{J}\right)}} \mathbb{E}\left[\mathbf{I}\left(\mathbf{y}, \mathbf{u}^{1}, \ldots, \mathbf{u}^{J}\right)\right] \\
& \leqslant q^{-\Omega(n N)}
\end{aligned}
$$

Before we proceed, we need a final bit of notation. For a good tuple $\left(\mathbf{u}^{1}, \ldots, \mathbf{u}^{J}\right)$ and every $1 \leqslant j \leqslant J$, define $T_{j}\left(\mathbf{u}^{1}, \ldots, \mathbf{u}^{J}\right) \subseteq[N]$ to be the set of positions $i$ such that $\mathbf{u}_{i}^{j}$ is not in the $\mathbb{F}_{q^{-}}$span of $\left\{\mathbf{u}_{i}^{1}, \ldots, \mathbf{u}_{i}^{j-1}\right\}$. (Here we view $\mathbb{F}_{Q}$ as the set of vectors from $\mathbb{F}_{q}^{k}$-recall that $\mathbb{F}_{q^{k}}$ is isomorphic to $\mathbb{F}_{q}^{k}$.) Note that since the tuple is good, $\left|T_{j}\left(\mathbf{u}^{1}, \ldots, \mathbf{u}^{J}\right)\right| \geqslant(1-R-\gamma) N$.

\footnotetext{
${ }^{6}$ We stress that we do not require that the $\mathbf{G}_{i}$ 's have rank $k$.
} 
Let $h=\rho n N$. Consider the following sequence of inequalities (where below we have suppressed the dependence of $T_{j}$ on $\left(\mathbf{u}^{1}, \ldots, \mathbf{u}^{J}\right)$ for clarity):

$$
\begin{aligned}
\mathbb{E}\left[X_{C}\right] & =\sum_{\mathbf{y} \in \mathbb{F}_{q}^{n N}} \sum_{\substack{\text { good } \\
\left(\mathbf{u}^{1}, \ldots, \mathbf{u}^{J}\right) \\
\in\left(C_{\text {out }}\right)^{J}}} \underset{\mathbf{G}}{\operatorname{Pr}}\left[\bigwedge_{j=1}^{J} w t\left(\mathbf{u}^{j} \mathbf{G}-\mathbf{y}\right) \leqslant h\right] \\
& \sum_{\mathbf{y} \in \mathbb{F}_{q}^{n N}} \sum_{\substack{\text { good } \\
\left(\mathbf{u}^{1}, \ldots, \mathbf{u}^{J}\right) \\
\in\left(C_{\text {out }}\right)^{J}}} \underset{\mathbf{G}}{\operatorname{Pr}}\left[\bigwedge_{j=1}^{J} w t_{T_{j}}\left(\mathbf{u}^{j} \mathbf{G}-\mathbf{y}\right) \leqslant h\right] \\
& =\sum_{\mathbf{y} \in \mathbb{F}_{q}^{n N}} \sum_{\substack{\text { good } \\
\left(\mathbf{u}^{1}, . ., \mathbf{u}^{J}\right) \\
\in\left(C_{\text {out }}\right)^{J}}} \prod_{j=1}^{J} \mathbf{G}\left[w t_{T_{j}}\left(\mathbf{u}^{j} \mathbf{G}-\mathbf{y}\right) \leqslant h\right]
\end{aligned}
$$

In the above (4) follows from the definition of the indicator variable. (15) follows from the simple fact that for every vector $\mathbf{u}$ of length $N$ and every $T \subseteq[N], w t_{T}(\mathbf{u}) \leqslant w t(\mathbf{u})$. ([6) follows from the subsequent argument. As all symbols corresponding to $T_{J}$ are good symbols, for every $i \in T_{J}$, the value of $\mathbf{u}_{i}^{J} \mathbf{G}_{i}$ is not in the $\mathbb{F}_{q}$-span of $\left\{\mathbf{u}_{i}^{1} \mathbf{G}_{i}, \ldots, \mathbf{u}_{i}^{J-1} \mathbf{G}_{i}\right\}$. Thus, by Lemma 5 and the fact that each of $\mathbf{G}_{1}, \ldots, \mathbf{G}_{N}$ are chosen independently (at random),

$$
\underset{\mathbf{G}}{\operatorname{Pr}}\left[\bigwedge_{j=1}^{J} w t_{T_{j}}\left(\mathbf{u}^{j} \mathbf{G}-\mathbf{y}\right) \leqslant h\right]=\underset{\mathbf{G}}{\operatorname{Pr}}\left[w t_{T_{J}}\left(\mathbf{u}^{J} \mathbf{G}-\mathbf{y}\right) \leqslant h\right] \cdot \underset{\mathbf{G}}{\operatorname{Pr}}\left[\bigwedge_{j=1}^{J-1} w t_{T_{j}}\left(\mathbf{u}^{j} \mathbf{G}-\mathbf{y}\right) \leqslant h\right] .
$$

Inductively applying the argument above gives ([6]). Further (where below we use $\bar{D}$ to denote $(1-R-\gamma) N)$

$$
\begin{aligned}
\mathbb{E}\left[X_{C}\right] \leqslant \sum_{\mathbf{y} \in \mathbb{F}_{q}^{n N}} \sum_{\operatorname{good}\left(\mathbf{u}^{1}, \ldots, \mathbf{u}^{J}\right) \in\left(C_{\text {out }}\right)^{J}} \prod_{j=1}^{J} q^{-n\left|T_{j}\right|\left(1-H_{q}\left(\frac{h}{n\left|T_{j}\right|}\right)\right)} \\
=\sum_{\mathbf{y} \in \mathbb{F}_{q}^{n N}} \sum_{\substack{\left(d_{1}, . ., d_{J}\right) \in\{\bar{D}, . ., N\}^{J} \operatorname{good}\left(\mathbf{u}^{1}, \ldots, \mathbf{u}^{J}\right) \in\left(C_{\text {out }}\right)^{J}, j=1 \\
\left|T_{1}\right|=d_{1}, \ldots,\left|T_{J}\right|=d_{J}}} \prod_{\substack{\left(d_{1}, \ldots, d_{J}\right) \\
\in\{\bar{D}, \ldots, N\}^{J}}} q^{-n d_{j}+n d_{j} H_{q}\left(\frac{h}{n d_{j}}\right)} \cdot \prod_{j=1}^{J N} q^{\max \left(d_{j}-(1-R) N+1,0\right)} \prod_{j=1}^{J} q^{-n d_{j}\left(1-H_{q}\left(\frac{h}{n d_{j}}\right)\right)} \\
\leqslant \sum_{\substack{\left(d_{1}, \ldots, d_{J}\right) \\
\in\{\bar{D}, \ldots, N\}^{J}}} q^{n N} \cdot q^{N J(J+1)} \cdot \prod_{j=1}^{J} Q^{d_{j}-(1-R-\gamma) N} \prod_{j=1}^{J} q^{-n d_{j}\left(1-H_{q}\left(\frac{h}{n d_{j}}\right)\right)} \\
=\sum_{\substack{\left(d_{1}, \ldots, d_{J}\right) \\
\in\{\bar{D}, \ldots, N\}^{J}}} \prod_{j=1}^{J} q^{-n d_{j}\left(1-H_{q}\left(\frac{h}{n d_{j}}\right)-r\left(1-\frac{(1-R-\gamma) N}{d_{j}}\right)-\frac{N}{J d_{j}}-\frac{N(J+1)}{n d_{j}}\right)} .
\end{aligned}
$$


(17) follows from (6) and Lemma [4 (8) follows from rearranging the summand and using the fact that the tuple is good (and hence $\left.d_{j} \geqslant(1-R-\gamma) N\right)$. (9) follows from the fact that there are $q^{n N}$ choices for $\mathbf{y}$ and Lemma $\mathbf{7} 7$ (10) follows from the fact that $d_{j}-(1-R) N+1 \leqslant d_{j}-(1-R-\gamma) N$ (for $N \geqslant 1 / \gamma)$ and that $d_{j} \geqslant(1-R-\gamma) N$. (11) follows by rearranging the terms.

Note that as long as $n \geqslant J(J+1)$, we have $\frac{N(J+1)}{n d} \leqslant \frac{N}{J d}$. Now (11) will imply (3) if we can show that for every $(1-R-\gamma) N \leqslant d \leqslant N$,

$$
\frac{h}{n d} \leqslant H_{q}^{-1}\left(1-r\left(1-\frac{(1-R-\gamma) N}{d}\right)-\frac{2 N}{J d}\right)-\delta,
$$

for $\delta=\varepsilon / 3$. By Lemma 8 (which is stated at the end of this section), as long as $J \geqslant 4 c_{q}^{\prime} /\left(\delta^{2}(1-R)\right.$ ) (and the conditions on $\gamma$ are satisfied), the above can be satisfied by picking

$$
h /(n N)=H_{q}^{-1}(1-r R)-3 \delta=\rho,
$$

as required. We now verify that the conditions on $\gamma$ in Lemma 8 are satisfied by picking $\gamma=\frac{4}{J r}$. Note that if we choose $J=4 c_{q}^{\prime} /\left(\delta^{2}(1-R)\right)$, we will have $\gamma=\frac{\delta^{2}(1-R)}{c_{q}^{\prime} r}$. Now, as $0<R<1$, we also have $\gamma \leqslant \delta^{2} /\left(r c_{q}^{\prime}\right)$. Finally, we show that $\gamma \leqslant(1-R) / 2$. Indeed

$$
\gamma=\frac{\delta^{2}(1-R)}{c_{q}^{\prime} r}=\frac{\varepsilon^{2}(1-R)}{9 c_{q}^{\prime} r} \leqslant \frac{\varepsilon(1-R)}{9 r}<\frac{\alpha_{q}(r)(1-R)}{9 r}<\frac{1-R}{2},
$$

where the first inequality follows from the facts that $c_{q}^{\prime} \geqslant 1$ and $\varepsilon \leqslant 1$. The second inequality follows from the assumption on $\varepsilon$. The third inequality follows from Lemma 2, As $J$ is in $\Theta\left(\frac{1}{\varepsilon^{2}(1-R)}\right)$ (and $\gamma$ is in $\Theta\left(\varepsilon^{2}(1-R) / r\right)$ ), we can choose $L=\left(N / \varepsilon^{4}\right)^{O\left(r^{2} \varepsilon^{-4}(1-R)^{-2} \log (q / R)\right)}$, as required.

We still need to argue that with high probability the rate of the code $C=C_{\text {out }} \circ\left(C_{i n}^{1}, \ldots, C_{i n}^{N}\right)$ is $r R$. One way to argue this would be to show that with high probability all of the generator matrices have full rank. However, this is not the case: in fact, with some non-negligible probability at least one of them will not have full rank. However, we claim that with high probability $C$ has distance $>0$, and thus is a subspace of dimension $r R n N$. The proof above in fact implies that with high probability $C$ has distance $\left(H_{q}^{-1}(1-r R)-\delta\right) n N$ for any small enough $\delta>0$. It is easy to see that to show that $C$ has distance at least $h$, it is enough to show that with high probability $\sum_{\mathbf{m} \in \mathbb{F}_{Q}^{K}} \mathbf{I}(\mathbf{0}, \mathbf{m})=0$. Note that this is a special case of our proof, with $J=1$ and $\mathbf{y}=\mathbf{0}$ and hence, with probability at least $1-q^{\Omega(n N)}$, the code $C$ has large distance.

The proof is thus complete, modulo the following lemma, which we prove next (following a similar argument in [16]).

Lemma 8. Let $q \geqslant 2$ be an integer, and $1 \leqslant n \leqslant N$ be integers. Let $0<r, R \leqslant 1$ be rationals and $\delta>0$ be a real such that $R \leqslant\left(\alpha_{q}(r)-\delta\right) / r$ and $\delta \leqslant \alpha_{q}(r)$, where $\alpha_{q}(r)$ is as defined in (1). Let $\gamma>0$ be a real such that $\gamma \leqslant \min \left(\frac{1-R}{2}, \frac{\delta^{2}}{c_{q}^{\prime} r}\right)$, where $c_{q}^{\prime}$ is the constant that depends only on $q$

\footnotetext{
${ }^{7}$ As the final code $C$ will be linear, it is sufficient to only look at received words that have Hamming weight at most $\rho n N$. However, this gives a negligible improvement to the final result and hence, we just bound the number of choices for $\mathbf{y}$ by $q^{n N}$.
} 
from Lemma 1. Then for all integers $J \geqslant \frac{4 c_{q}^{\prime}}{\delta^{2}(1-R)}$ and $h \leqslant\left(H_{q}^{-1}(1-r R)-2 \delta\right) n N$ the following is satisfied. For every integer $(1-R-\gamma) N \leqslant d \leqslant N$,

$$
\frac{h}{n d} \leqslant H_{q}^{-1}\left(1-r\left(1-\frac{N(1-R-\gamma)}{d}\right)-\frac{2 N}{J d}\right) .
$$

Proof. Using the fact $H_{q}^{-1}$ is an increasing function, (12) is satisfied if for every $d^{*} \leqslant d \leqslant N$ (where $\left.d^{*}=(1-R-\gamma) N\right)$ :

$$
\frac{h}{n N} \leqslant\left(\frac{d}{N}\right) \cdot H_{q}^{-1}\left(1-r\left(1-\frac{N(1-R-\gamma)}{d}\right)-\frac{2 N}{d^{*} J}\right) .
$$

Define a new variable $\theta=1-N(1-R-\gamma) / d$. Note that as $d^{*}=(1-R-\gamma) N \leqslant d \leqslant N$, $0 \leqslant \theta \leqslant R+\gamma$. Also $d / N=(1-R-\gamma)(1-\theta)^{-1}$. Thus, the above inequality would be satisfied if

$$
\frac{h}{n N} \leqslant(1-R-\gamma) \min _{0 \leqslant \theta \leqslant R+\gamma}\left\{(1-\theta)^{-1} H_{q}^{-1}\left(1-r \theta-\frac{2}{(1-R-\gamma) J}\right)\right\} .
$$

Again using the fact that $H_{q}^{-1}$ is an increasing function along with the fact that $\gamma \leqslant(1-R) / 2$, we get that the above is satisfied if

$$
\frac{h}{n N} \leqslant(1-R-\gamma) \min _{0 \leqslant \theta \leqslant R+\gamma}\left\{(1-\theta)^{-1} H_{q}^{-1}\left(1-r \theta-\frac{4}{(1-R) J}\right)\right\} .
$$

By Lemma 1] if $J \geqslant \frac{4 c_{q}^{\prime}}{\delta^{2}(1-R)}$, then $8 H_{q}^{-1}\left(1-r \theta-\frac{4}{(1-R) J}\right) \geqslant H_{q}^{-1}(1-r \theta)-\delta$. Since for every $0 \leqslant \theta \leqslant R+\gamma,(1-R-\gamma)(1-\theta)^{-1} \delta \leqslant \delta$, the above equation would be satisfied if

$$
\frac{h}{n N} \leqslant(1-R-\gamma) \min _{0<\theta \leqslant R+\gamma} f_{r, q}(\theta)-\delta
$$

Note that by the assumptions $\gamma \leqslant \delta^{2} /\left(r c_{q}^{\prime}\right) \leqslant \delta / r$ (as $\delta \leqslant 1$ and $c_{q}^{\prime} \geqslant 1$ ) and $R \leqslant\left(\alpha_{q}(r)-\delta\right) / r$, we have $R+\gamma \leqslant \alpha_{q}(r) / r$. Thus, by using Lemma [3] we get that $(1-R-\gamma) \min _{0<\theta \leqslant R+\gamma} f_{r, q}(\theta)=$ $H_{q}^{-1}(1-r R-r \gamma)$. By Lemma 1] the facts that $\gamma \leqslant \delta^{2} /\left(r c_{q}^{\prime}\right)$ and $H_{q}^{-1}$ is increasing, we have $H_{q}^{-1}(1-r R-r \gamma) \geqslant H_{q}^{-1}(1-r R)-\delta$. This implies that (12) is satisfied if $h /(n N) \leqslant H_{q}^{-1}(1-r R)-2 \delta$, as desired.

\section{$5 \quad$ List Decodability of Random Concatenated Codes}

In this section, we will look at the list decodability of concatenated codes when both the outer code and the inner codes are (independent) random linear codes. The following is the main result of this section.

Theorem 3. Let $q$ be a prime power and let $0<r \leqslant 1$ be an arbitrary rational. Let $0<\varepsilon<\alpha_{q}(r)$ be an arbitrary real, where $\alpha_{q}(r)$ is as defined in (1), and $0<R \leqslant\left(\alpha_{q}(r)-\varepsilon\right) / r$ be a rational. Let $k, n, K, N \geqslant 1$ be large enough integers such that $k=r n$ and $K=R N$. Let $C_{\text {out }}$ be a random linear

${ }^{8}$ We also use the fact that $H_{q}^{-1}$ is increasing. 
code over $\mathbb{F}_{q^{k}}$ that is generated by a random $K \times N$ matrix over $\mathbb{F}_{q^{k}}$. Let $C_{i n}^{1}, \ldots, C_{i n}^{N}$ be random linear codes over $\mathbb{F}_{q}$, where $C_{i n}^{i}$ is generated by a random $k \times n$ matrix $\mathbf{G}_{i}$ and the random choices for $C_{\text {out }}, \mathbf{G}_{1}, \ldots, \mathbf{G}_{N}$ are all independent. Then the concatenated code $C=C_{\text {out }} \circ\left(C_{\text {in }}^{1}, \ldots, C_{\text {in }}^{N}\right)$ is $a\left(H_{q}^{-1}(1-R r)-\varepsilon, q^{O\left(\frac{r n}{\varepsilon^{2}(1-R)}\right)}\right)$-list decodable code with probability at least $1-q^{-\Omega(n N)}$ over the choices of $C_{\text {out }}, \mathbf{G}_{1}, \ldots, \mathbf{G}_{N}$. Further, with high probability, $C$ has rate $r$.

The intuition behind Theorem 3 is the following. W.h.p., a random code has a weight distribution and list recoverability properties very similar to those of folded Reed-Solomon codes. That is, Lemmas [ 6] and [7hold whp for random $C_{\text {out }}$. However, we will prove Theorem 3 in a slightly different manner than the proof of Theorem 2 as it gives a better bound on the list size (see Remark 2 for a more quantitative comparison). In the rest of this section, we will prove Theorem 3 .

Define $Q=q^{k}$. Let $L$ be the worst-case list size that we are shooting for (we will fix its value at the end). The first observation is that any $L+1$-tuple of messages $\left(\mathbf{m}^{1}, \ldots, \mathbf{m}^{L+1}\right) \in\left(\mathbb{F}_{Q}^{K}\right)^{L+1}$ contains at least $J=\left\lceil\log _{Q}(L+1)\right\rceil$ many messages that are linearly independent over $\mathbb{F}_{Q}$. Thus, to prove the theorem it suffices to show that with high probability, no Hamming ball over $\mathbb{F}_{q}^{n N}$ of radius $\left(H_{q}^{-1}(1-r R)-\varepsilon\right) n N$ contains a $J$-tuple of codewords $\left(C\left(\mathbf{m}^{1}\right), \ldots, C\left(\mathbf{m}^{J}\right)\right)$, where $\mathbf{m}^{1}, \ldots, \mathbf{m}^{J}$ are linearly independent over $\mathbb{F}_{Q}$.

Define $\rho=H_{q}^{-1}(1-R r)-\varepsilon$. For every $J$-tuple of linearly independent messages $\left(\mathbf{m}^{1}, \ldots, \mathbf{m}^{J}\right) \in$ $\left(\mathbb{F}_{Q}^{K}\right)^{J}$ and received word $\mathbf{y} \in \mathbb{F}_{q}^{n N}$, define an indicator random variable $\mathbf{I}\left(\mathbf{y}, \mathbf{m}^{1}, \ldots, \mathbf{m}^{J}\right)$ as follows. $\mathbf{I}\left(\mathbf{y}, \mathbf{m}^{1}, \ldots, \mathbf{m}^{J}\right)=1$ if and only if for every $1 \leqslant j \leqslant J, w t\left(C\left(\mathbf{m}^{j}\right)-\mathbf{y}\right) \leqslant \rho n N$. That is, it captures the bad event that we want to avoid. Define

$$
X_{C}=\sum_{\mathbf{y} \in \mathbb{F}_{q}^{n N}} \sum_{\left(\mathbf{m}^{1}, \ldots, \mathbf{m}^{J}\right) \in \operatorname{Ind}(Q, K, J)} \mathbf{I}\left(\mathbf{y}, \mathbf{m}^{1}, \ldots, \mathbf{m}^{J}\right)
$$

where $\operatorname{Ind}(Q, K, J)$ denotes the collection of subsets of $\mathbb{F}_{Q}$-linearly independent vectors from $\mathbb{F}_{Q}^{K}$ of size $J$. We want to show that with high probability $X_{C}=0$. By Markov's inequality, the theorem would follow if we can show that:

$$
\mathbb{E}\left[X_{C}\right]=\sum_{\mathbf{y} \in \mathbb{F}_{q}^{n N}} \sum_{\left(\mathbf{m}^{1}, \ldots, \mathbf{m}^{J}\right) \in \operatorname{Ind}(Q, K, J)} \mathbb{E}\left[\mathbf{I}\left(\mathbf{y}, \mathbf{m}^{1}, \ldots, \mathbf{m}^{J}\right)\right] \text { is at most } q^{-\Omega(n N)} .
$$

Note that the number of distinct possibilities for $\mathbf{y}, \mathbf{m}^{1}, \ldots, \mathbf{m}^{J}$ is upper bounded by $q^{n N} \cdot Q^{R N J}=$ $q^{n N(1+r R J)}$. Fix some arbitrary choice of $\mathbf{y}, \mathbf{m}^{1}, \ldots, \mathbf{m}^{J}$. To prove (13), we will show that

$$
q^{n N(1+r R J)} \cdot \mathbb{E}\left[\mathbf{I}\left(\mathbf{y}, \mathbf{m}^{1}, \ldots, \mathbf{m}^{J}\right)\right] \leqslant q^{-\Omega(n N)} .
$$

Before we proceed, we need some more notation. Given vectors $\mathbf{u}^{1}, \ldots, \mathbf{u}^{J} \in \mathbb{F}_{Q}^{N}$, we define $\mathbf{Z}\left(\mathbf{u}^{1}, \ldots, \mathbf{u}^{J}\right)=\left(Z_{1}, \ldots, Z_{N}\right)$ as follows. For every $1 \leqslant i \leqslant N, Z_{i} \subseteq[J]$ denotes the largest subset such that the elements $\left(u_{i}^{j}\right)_{j \in Z_{i}}$ are linearly independent over $\mathbb{F}_{q}$ (in case of a tie choose the lexically first such set), where $\mathbf{u}^{j}=\left(u_{1}^{j}, \ldots, u_{N}^{j}\right)$. If $u_{i}^{j} \in Z_{i}$ then we will call $u_{i}^{j}$ a good symbol. Note that a good symbol is always non-zero. We will also define another partition of all the good symbols, $\mathbf{T}\left(\mathbf{u}^{1}, \ldots, \mathbf{u}^{J}\right)=\left(T_{1}, \ldots, T_{J}\right)$ by setting $T_{j}=\left\{i \mid j \in Z_{i}\right\}$ for $1 \leqslant j \leqslant J$. 
Since $\mathbf{m}^{1}, \ldots, \mathbf{m}^{J}$ are linearly independent over $\mathbb{F}_{Q}$, the corresponding codewords in $C_{\text {out }}$ are distributed uniformly and independently in $\mathbb{F}_{Q}^{N}$. In other words, for any fixed $\left(\mathbf{u}^{1}, \ldots, \mathbf{u}^{J}\right) \in\left(\mathbb{F}_{Q}^{N}\right)^{J}$,

$$
\operatorname{Pr}_{C_{\text {out }}}\left[\bigwedge_{j=1}^{J} C_{\text {out }}\left(\mathbf{m}^{j}\right)=\mathbf{u}^{j}\right]=Q^{-N J}=q^{-r n N J} .
$$

Recall that we denote the (random) generator matrices for the inner code $C_{i n}^{i}$ by $\mathbf{G}_{i}$ for every $1 \leqslant i \leqslant N$. Also note that every $\left(\mathbf{u}^{1}, \ldots, \mathbf{u}^{J}\right) \in\left(\mathbb{F}_{Q}^{N}\right)^{J}$ has a unique $\mathbf{Z}\left(\mathbf{u}^{1}, \ldots, \mathbf{u}^{J}\right)$. In other words, the $2^{N J}$ choices of $\mathbf{Z}$ partition the tuples in $\left(\mathbb{F}_{Q}^{N}\right)^{J}$.

Let $h=\rho n N$. Consider the following calculation (where the dependence of $\mathbf{Z}$ and $\mathbf{T}$ on $\mathbf{u}^{1}, \ldots, \mathbf{u}^{J}$ have been suppressed for clarity):

$$
\begin{aligned}
\mathbb{E}\left[\mathbf{I}\left(\mathbf{y}, \mathbf{m}^{1}, \ldots, \mathbf{m}^{J}\right)\right]= & \sum_{\left(\mathbf{u}^{1}, \ldots, \mathbf{u}^{J}\right) \in\left(\mathbb{F}_{Q}^{N}\right)^{J}} \operatorname{Pr}_{\mathbf{G}^{\prime}=\left(\mathbf{G}_{1}, \ldots, \mathbf{G}_{N}\right)}\left[\bigwedge_{j=1}^{J} w t\left(\mathbf{u}^{j} \mathbf{G}-\mathbf{y}\right) \leqslant h\right] \\
& \cdot \operatorname{Pr}_{C_{\text {out }}}\left[\bigwedge_{j=1}^{J} C_{\text {out }}\left(\mathbf{m}^{j}\right)=\mathbf{u}^{j}\right] \\
= & q^{-r n N J} \sum_{\left(\mathbf{u}^{1}, \ldots, \mathbf{u}^{J}\right) \in\left(\mathbb{F}_{Q}^{N}\right)^{J}} \operatorname{Pr}_{\mathbf{G}=\left(\mathbf{G}_{1}, \ldots, \mathbf{G}_{N}\right)}\left[\bigwedge_{j=1}^{J} w t\left(\mathbf{u}^{j} \mathbf{G}-\mathbf{y}\right) \leqslant h\right] \\
\leqslant & q^{-r n N J} \sum_{\left(\mathbf{u}^{1}, \ldots, \mathbf{u}^{J}\right) \in\left(\mathbb{F}_{Q}^{N}\right)^{J}} \operatorname{Pr}_{\mathbf{G}=\left(\mathbf{G}_{1}, \ldots, \mathbf{G}_{N}\right)}\left[\bigwedge_{j=1}^{J} w t_{T_{j}}\left(\mathbf{u}^{j} \mathbf{G}-\mathbf{y}\right) \leqslant h\right] \\
= & q^{-r n N J} \sum_{\left(\mathbf{u}^{1}, \ldots, \mathbf{u}^{J}\right) \in\left(\mathbb{F}_{Q}^{N}\right)^{J}} \prod_{j=1}^{J} \operatorname{Pr}_{\mathbf{G}}\left[w t_{T_{j}}\left(\mathbf{u}^{j} \mathbf{G}-\mathbf{y}\right) \leqslant h\right]
\end{aligned}
$$

In the above (16) follows from the fact that the (random) choices for $C_{\text {out }}$ and $\mathbf{G}=\left(\mathbf{G}_{1}, \ldots, \mathbf{G}_{N}\right)$ are all independent. (17) follows from (15). (18) follows from the simple fact that for every $\mathbf{y} \in\left(\mathbb{F}_{q}^{n}\right)^{N}$ and $T \subseteq[N], w t_{T}(\mathbf{y}) \leqslant w t(\mathbf{y})$. (19) follows from the same argument used to prove (6).

Further,

$$
\begin{aligned}
\mathbb{E}\left[\mathbf{I}\left(\mathbf{y}, \mathbf{m}^{1}, \ldots, \mathbf{m}^{J}\right)\right] & =\sum_{\substack{\left(\mathbf{u}^{1}, \ldots, \mathbf{u}^{J}\right) \in\left(\mathbb{F}_{Q}^{N}\right)^{J} \\
j=1}} \prod^{J} q^{-r n N} \cdot \operatorname{Pr}_{\mathbf{G}}\left[w t_{T_{j}}\left(\mathbf{u}^{j} \mathbf{G}-\mathbf{y}\right) \leqslant h\right] \\
& =\sum_{\substack{\left(d_{1}, \ldots, d_{J}\right) \in\{0, \ldots, N\}^{J} \\
\left(\mathbf{u}^{1}, \ldots, \mathbf{u}^{J}\right) \in\left(\mathbb{F}_{Q}^{N}\right)^{J}, j=1}} \prod_{\left(T_{1}\left|=d_{1}, \ldots,\right| T_{J} \mid d_{J}\right)}^{J} \frac{\operatorname{Pr}_{\mathbf{G}}\left[w t_{T_{j}}\left(\mathbf{u}^{j} \mathbf{G}-\mathbf{y}\right) \leqslant h\right]}{q^{r n N}} \\
& \leqslant \sum_{\substack{\left(d_{1}, \ldots, d_{J}\right) \\
\in\{0, \ldots, N\}^{J}}} q^{J N+(r n+J) \sum_{j=1}^{J} d_{j}} \prod_{\substack{j=1,\left|T_{j}\right|=d_{j}}}^{J} \frac{\operatorname{Pr}_{\mathbf{G}}\left[w t_{T_{j}}\left(\mathbf{u}^{j} \mathbf{G}-\mathbf{y}\right) \leqslant h\right]}{q^{r n N}}
\end{aligned}
$$




$$
=\sum_{\left(d_{1}, \ldots, d_{J}\right) \in\{0, \ldots, N\}^{J}} \prod_{\substack{j=1,\left|T_{j}\right|=d_{j}}}^{J} \frac{\operatorname{Pr}_{\mathbf{G}}\left[w t_{T_{j}}\left(\mathbf{u}^{j} \mathbf{G}-\mathbf{y}\right) \leqslant h\right]}{q^{n\left(-r\left(d_{j}-N\right)-\frac{J d_{j}}{n}-\frac{N}{n}\right)}}
$$

In the above (20), (21), (23) follow from rearranging and grouping the summands. (22) uses the following argument. Given a fixed $\mathbf{Z}=\left(Z_{1}, \ldots, Z_{N}\right)$, the number of tuples $\left(\mathbf{u}^{1}, \ldots, \mathbf{u}^{J}\right)$ such that $\mathbf{Z}\left(\mathbf{u}^{1}, \ldots, \mathbf{u}^{J}\right)=\mathbf{Z}$ is at most $U=\prod_{i=1}^{N} q^{\left|Z_{i}\right| k} \cdot q^{\left|Z_{i}\right|\left(J-\left|Z_{i}\right|\right)}$, where the $q^{\left|Z_{i}\right| k}$ is an upper bound on the number of $\left|Z_{i}\right|$ linearly independent vectors from $\mathbb{F}_{q}^{k}$ and $q^{\left|Z_{i}\right|\left(J-\left|Z_{i}\right|\right)}$ follows from the fact that every bad symbol $\left\{u_{i}^{j}\right\}_{j \notin Z_{i}}$ has to take a value that is a linear combination of the symbols $\left\{u_{i}^{j}\right\}_{j \in Z_{i}}$. Now $U \leqslant \prod_{i=1}^{N} q^{\left|Z_{i}\right|(k+J)}=q^{(k+J) \sum_{i=1}^{N}\left|Z_{i}\right|}=q^{(k+J) \sum_{j=1}^{J}\left|T_{j}\right|}$. Finally, recall that there are $2^{J N} \leqslant q^{J N}$ distinct choices for $\mathbf{Z}$.

(23) implies the following

$$
q^{n N(1+r R J)} \cdot \mathbb{E}\left[\mathbf{I}\left(\mathbf{y}, \mathbf{m}^{1}, \ldots, \mathbf{m}^{J}\right)\right] \leqslant \sum_{\left(d_{1}, \ldots, d_{J}\right) \in\{0, \ldots, N\}^{J}} \prod_{j=1}^{J} E_{j}
$$

where

$$
E_{j}=q^{-n\left(-r\left(d_{j}-N(1-R)\right)-\frac{N}{J}-\frac{J d_{j}}{n}-\frac{N}{n}\right)} \cdot \operatorname{Pr}_{\mathbf{G}}\left[w t_{T_{j}}\left(\mathbf{u}_{j} \mathbf{G}-\mathbf{y}\right) \leqslant h\right]
$$

We now proceed to upper bound $E_{j}$ by $q^{-\Omega(n N / J)}$ for every $1 \leqslant j \leqslant J$. Note that this will imply the claimed result as there are at most $(N+1)^{J}=q^{o(n N)}$ choices for different values of $d_{j}$ 's.

We first start with the case when $d_{j}<d^{*}$, where

$$
d^{*}=N(1-R-\gamma)
$$

for some parameter $0<\gamma<1-R$ to be defined later (note that we did not have to deal with this case in the proof of Theorem 2 2$)$. In this case we use the fact that $\operatorname{Pr}_{\mathbf{G}}\left[w t_{T_{j}}\left(\mathbf{u}_{j} \mathbf{G}-\mathbf{y}\right) \leqslant h\right] \leqslant 1$. Thus, we would be done if we can show that

$$
\frac{1}{N}\left(r\left(d_{j}-N(1-R)\right)+\frac{N}{J}+\frac{J d_{i}}{n}+\frac{N}{n}\right) \leqslant-\delta^{\prime}<0,
$$

for some $\delta^{\prime}>0$ that we will choose soon. The above would be satisfied if

$$
\frac{d_{j}}{N}<(1-R)-\frac{1}{r}\left(\frac{1}{J}+\frac{J d_{j}}{n N}+\frac{1}{n}\right)-\frac{\delta^{\prime}}{r},
$$

which is satisfied if we choose $\gamma>\frac{2}{r}\left(\frac{1}{J}+\frac{J d_{j}}{n N}+\frac{1}{n}\right)+\frac{\delta^{\prime}}{r}$ as $d_{j}<d^{*}$. Note that if $n>J\left(\frac{J d_{j}}{N}+1\right)$ and if we set $\delta^{\prime}=\frac{1}{J}$, it is enough to choose $\gamma=\frac{4}{J r}$.

We now turn our attention to the case when $d_{j} \geqslant d^{*}$. The arguments are very similar to those employed in the proof of Theorem 2 In this case, by Lemma 4 we have

$$
E_{j} \leqslant q^{-n d_{j}\left(1-H_{q}\left(\frac{h}{n d_{j}}\right)-r\left(1-\frac{N(1-R)}{d_{j}}\right)-\frac{N}{d_{j} J}-\frac{J}{n}-\frac{N}{n d_{j}}\right)} .
$$


The above implies that we can show that $E_{j}$ is $q^{-\Omega(n N(1-R-\gamma))}$ provided we show that for every $d^{*} \leqslant d \leqslant N$,

$$
\frac{h}{n d} \leqslant H_{q}^{-1}\left(1-r\left(1-\frac{N(1-R)}{d}\right)-\frac{N}{d J}-\frac{J}{n}-\frac{N}{n d}\right)-\delta,
$$

for $\delta=\varepsilon / 3$. Now if $n \geqslant 2 J^{2}$, then both $\frac{J}{n} \leqslant \frac{N}{2 J d}$ and $\frac{N}{n d} \leqslant \frac{N}{2 J d}$. In other words, $\frac{J}{n}+\frac{N}{n d} \leqslant \frac{N}{J d}$. Using the fact that $H_{q}^{-1}$ is increasing, the above is satisfied if

$$
\frac{h}{n d} \leqslant H_{q}^{-1}\left(1-r\left(1-\frac{N(1-R-\gamma)}{d}\right)-\frac{2 N}{d J}\right)-\delta
$$

As in the proof of Theorem 2 as long as $J \geqslant 4 c_{q}^{\prime} /\left(\delta^{2}(1-R)\right)$, by Lemma 8 the above can be satisfied by picking

$$
\frac{h}{n N}=H_{q}^{-1}(1-r R)-3 \delta=\rho
$$

as required.

Note that $J=O\left(\frac{1}{(1-R) \varepsilon^{2}}\right)$, which implies $L=Q^{O\left(1 /\left((1-R) \varepsilon^{2}\right)\right.}$ as claimed in the statement of the theorem. Again using the same argument used in the proof of Theorem 2 it can be shown that with high probability the rate of the code $C=C_{\text {out }} \circ\left(C_{i n}^{1}, \ldots, C_{i n}^{N}\right)$ is $r R$. The proof is complete.

Remark 2. The proof of Theorem 3 does not use the list recoverability property of the outer code directly. The idea of using list recoverability to argue independence can also be used to prove Theorem [3. That is, first show that with good probability, a random linear outer code will have good list recoverability. Then the argument in previous section can be used to prove Theorem 3 However, this gives worse parameters than the proof above. In particular, by a straightforward application of the probabilistic method, one can show that a random linear code of rate $R$ over $\mathbb{F}_{Q}$ is $\left(R+\gamma, \ell, Q^{\ell / \gamma}\right)$-list recoverable [5, Sec 9.3.2]. In proof of Theorem 2, $\ell$ is roughly $q^{J}$, where $J$ is roughly $1 / \varepsilon^{2}$. Thus, if we used the arguments in the proof of Theorem 2 we would be able to prove Theorem 3 but with lists of size of $Q^{q^{O\left(\varepsilon^{-2}(1-R)^{-1}\right)}}$, which is worse than the list size of $Q^{O\left(\varepsilon^{-2}(1-R)^{-1}\right)}$ guaranteed by Theorem 3 .

Remark 3. In a typical use of concatenated codes, the block lengths of the inner and outer codes satisfy $n=\Theta(\log N)$, in which case the concatenated code of Theorem 3 is list decodable with lists of size $N^{O\left(\varepsilon^{-2}(1-R)^{-1}\right)}$. However, the proof of Theorem 3 also works with smaller $n$. In particular as long as $n$ is at least $3 J^{2}$, the proof of Theorem 3 goes through. Thus, with $n$ in $\Theta\left(J^{2}\right)$, one can get concatenated codes that are list decodable up to the list-decoding capacity with lists of size $q^{O\left(\varepsilon^{-6}(1-R)^{-3}\right)}$.

\section{Open Questions}

In this work, we have shown that the family of concatenated codes is rich enough to contain codes that are list decodable up to the Hamming bound. But realizing the full potential of concatenated codes and achieving the Hamming bound (or even substantially improving upon the Blokh-Zyablov bound [1]) with explicit codes and polynomial time decoding remains a huge challenge. Achieving 
an explicit construction even without the requirement of an efficient list-decoding algorithm (but only good combinatorial list-decodability properties) is itself wide open.

The difficulty with explicit constructions is that we do not have any handle on the structure of inner codes that lead to concatenated codes with the required properties. In fact, we do not know of any efficient algorithm to even verify that a given set of inner codes will work, so even a Las Vegas construction appears difficult (a similar situation holds for binary codes meeting the Gilbert-Varshamov trade-off between rate and relative distance).

\section{References}

[1] E. L. Blokh and Victor V. Zyablov. Existence of linear concatenated binary codes with optimal correcting properties. Prob. Peredachi Inform., 9:3-10, 1973.

[2] Ilya I. Dumer. Concatenated codes and their multilevel generalizations. In V. S. Pless and W. C. Huffman, editors, Handbook of Coding Theory, volume 2, pages 1911-1988. North Holland, 1998.

[3] Peter Elias. Error-correcting codes for list decoding. IEEE Transactions on Information Theory, 37:5-12, 1991.

[4] G. David Forney. Concatenated Codes. MIT Press, Cambridge, MA, 1966.

[5] Venkatesan Guruswami. List decoding of error-correcting codes. Number 3282 in Lecture Notes in Computer Science. Springer, 2004.

[6] Venkatesan Guruswami, Johan Håstad, and Swastik Kopparty. On the list-decodability of random linear codes. arXiv:1001.1386, January 2010.

[7] Venkatesan Guruswami, Johan Håstad, Madhu Sudan, and David Zuckerman. Combinatorial bounds for list decoding. IEEE Transactions on Information Theory, 48(5):1021-1035, 2002.

[8] Venkatesan Guruswami and Piotr Indyk. Expander-based constructions of efficiently decodable codes. In Proceedings of the 42nd Annual IEEE Symposium on Foundations of Computer Science, pages 658-667, 2001.

[9] Venkatesan Guruswami and Atri Rudra. Limits to list decoding Reed-Solomon codes. IEEE Transactions on Information Theory, 52(8):3642-3649, August 2006.

[10] Venkatesan Guruswami and Atri Rudra. Explicit codes achieving list decoding capacity: Errorcorrection with optimal redundancy. IEEE Transactions on Information Theory, 54(1):135150, January 2008.

[11] Venkatesan Guruswami and Atri Rudra. Better binary list-decodable codes via multilevel concatenation. IEEE Transactions on Information Theory, 55(1):19-26, January 2009.

[12] F. J. MacWilliams and Neil J. A. Sloane. The Theory of Error-Correcting Codes. Elsevier/North-Holland, Amsterdam, 1981. 
[13] Jørn Justesen. A class of constructive asymptotically good algebraic codes. IEEE Transactions on Information Theory, 18:652-656, 1972.

[14] Atri Rudra. List Decoding and Property Testing of Error Correcting Codes. PhD thesis, University of Washington, 2007.

[15] Michael Sipser and Daniel Spielman. Expander codes. IEEE Transactions on Information Theory, 42(6):1710-1722, 1996.

[16] Christian Thommesen. The existence of binary linear concatenated codes with Reed-Solomon outer codes which asymptotically meet the Gilbert-Varshamov bound. IEEE Transactions on Information Theory, 29(6):850-853, November 1983.

[17] Christian Thommesen. Error-correcting capabilities of concatenated codes with mds outer codes on memoryless channels with maximum- likelihood decoding. IEEE Transactions on Information Theory, 33(5):632-640, 1987.

[18] Victor V. Zyablov and Mark S. Pinsker. List cascade decoding. Problems of Information Transmission, 17(4):29-34, 1981 (in Russian); pp. 236-240 (in English), 1982.

\section{A Proof of Lemma 3}

Proof. The proof follows from the subsequent geometric interpretations of $f_{x, q}(\cdot)$ and $\alpha_{q}(\cdot)$. See Figure 1 for a pictorial illustration of the arguments used in this proof (for $q=2$ ).

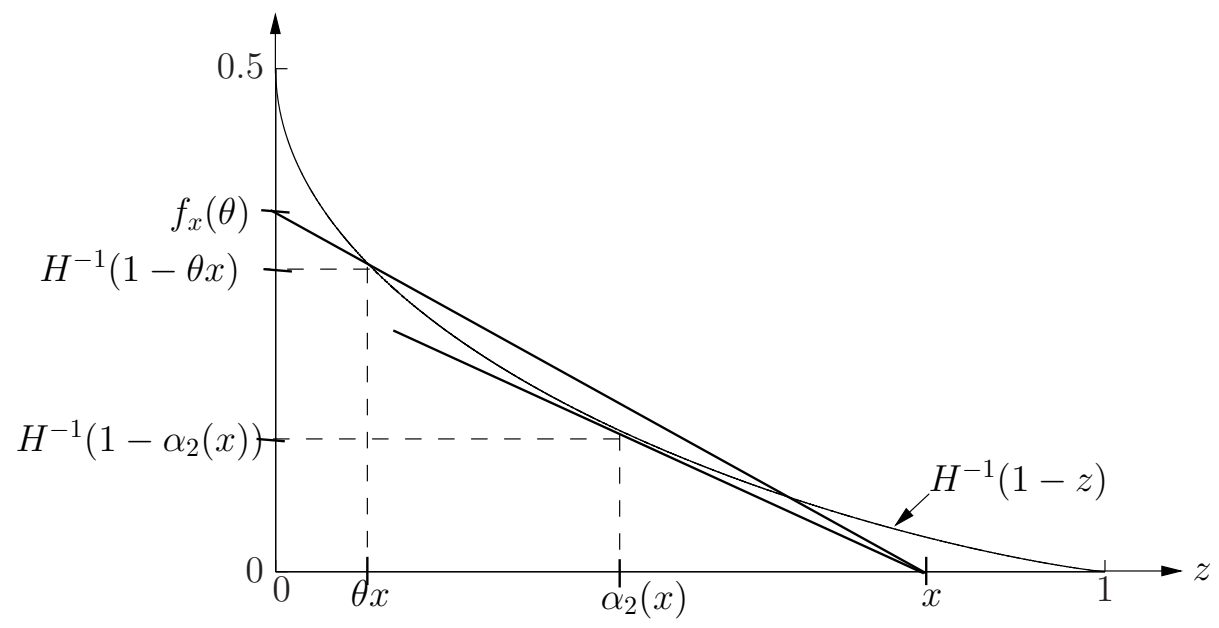

Figure 1: Geometric interpretations of functions $\alpha_{2}(\cdot)$ and $f_{x, 2}(\cdot)$.

First, we claim that for any $0 \leqslant z_{0} \leqslant 1, \alpha_{q}\left(z_{0}\right)$ satisfies the following property: the line segment between $\left(\alpha_{q}\left(z_{0}\right), H_{q}^{-1}\left(1-\alpha_{q}\left(z_{0}\right)\right)\right)$ and $\left(z_{0}, 0\right)$ is tangent to the curve $H_{q}^{-1}(1-z)$ at $\alpha_{q}\left(z_{0}\right)$.

Thus, we need to show that

$$
\frac{-H_{q}^{-1}\left(1-\alpha_{q}\left(z_{0}\right)\right)}{z_{0}-\alpha_{q}\left(z_{0}\right)}=\left(H_{q}^{-1}\right)^{\prime}\left(1-\alpha_{q}\left(z_{0}\right)\right) .
$$


One can check that $\left(H_{q}^{-1}\right)^{\prime}(1-x)=\frac{-1}{H_{q}^{\prime}\left(H_{q}^{-1}(1-x)\right)}=\frac{-1}{\log _{q}(q-1)-\log _{q}\left(H_{q}^{-1}(1-x)\right)+\log _{q}\left(1-H_{q}^{-1}(1-x)\right)}$. Now,

$$
\begin{aligned}
z_{0}-\alpha_{q}\left(z_{0}\right) & =z_{0}-1+\left(1-q^{z_{0}-1}\right) \log _{q}(q-1)-\left(1-q^{z_{0}-1}\right) \log _{q}\left(1-q^{z_{0}-1}\right)-q^{z_{0}-1}\left(z_{0}-1\right) \\
& =\left(1-q^{z_{0}-1}\right) \cdot\left(\log _{q}(q-1)-\log _{q}\left(1-q^{z_{0}-1}\right)+z_{0}-1\right) \\
& =H_{q}^{-1}\left(1-\alpha_{q}\left(z_{0}\right)\right) \cdot\left(\log _{q}(q-1)-\log _{q}\left(H_{q}^{-1}\left(1-\alpha_{q}\left(z_{0}\right)\right)+\log _{q}\left(1-H_{q}^{-1}\left(1-\alpha_{q}\left(z_{0}\right)\right)\right)\right)\right. \\
& =\frac{-H_{q}^{-1}\left(1-\alpha_{q}\left(z_{0}\right)\right)}{\left(H_{q}^{-1}\right)^{\prime}\left(1-\alpha_{q}\left(z_{0}\right)\right)}
\end{aligned}
$$

which proves (24) (where we have used the expression for $\alpha_{q}(z)$ and $\left(H_{q}^{-1}\right)^{\prime}(1-z)$ and the fact that $\left.1-q^{z-1}=H_{q}^{-1}\left(1-\alpha_{q}(z)\right)\right)$.

We now claim that $f_{x, q}(\theta)$ is the intercept of the line segment through $(x, 0)$ and $\left(\theta x, H_{q}^{-1}(1-\theta x)\right)$ on the " $y$-axis." Indeed, the " $y$-coordinate" increases by $H_{q}^{-1}(1-\theta x)$ in the line segment from $x$ to $\theta x$. Thus, when the line segment crosses the " $y$-axis," it would cross at an intercept of $1 /(1-\theta)$ times the gain going from $x$ to $\theta x$. The lemma follows from the fact that the function $H_{q}^{-1}(1-r)$ is a decreasing (strictly) convex function of $r$ and thus, the minimum of $f_{x, q}(\theta)$ would occur at $\theta=y$ provided $y x \leqslant \alpha_{q}(x)$. 